\title{
Real-time Monitoring of Biomarkers: Current Status and Future Perspectives
}

\author{
Se-Hwan Paek (iD* \\ Received: 20 January, 2020 / Accepted: 4 February, 2020 / Published online: 5 March, 2020 \\ (C) The Korean BioChip Society and Springer 2020
}

\section{Keywords: Real-time monitoring, Biomarkers, Liquid biopsy, Unattended healthcare, Unmet needs}

Real-time monitoring is a technique that can be accomplished in the future by continuous or high-frequency analyses of biomarkers indicating disorders (e.g., acute myocardial infarction) in an unattended manner, which eventually will occur in wearable or implantable biosensor formats (refer to Figure 1) ${ }^{1}$. This technique can enable early diagnosis and prognosis for target diseases, and even provide information related to the disease in progress and therapeutic effect in personalized medicine. The biochemical signs related to a disease will be combined with real-time physical signals such as cardiac impulse and pulsation, significantly increasing diagnostic accuracy $^{2}$. A great number of disease-related biomarkers have been clinically validated, and various types of analytes from simple molecules, such as ions and metabolites, to large, complex markers, such as proteins and cells, are available. To achieve unattended care through real-time monitoring, unmet technical demands remained are as follows: 1) (semi-)continuous drawings of venous blood without pains and infection, 2) (semi-)continuous separation of blood cells, 3) recyclable immunosorbent, 4) miniaturized, possibly, label-free sensor system, 5) in situ baseline correction algorithm, and 6) secure data transmission.

Recently, liquid biopsy has started to emerge for, in particular, cancer diagnosis via detection of circulating DNA, tumor cells, and exosomes in body fluids, instead of removing a piece of tissue for examination ${ }^{3}$. For these markers, as they are usually present in extremely low concentrations in samples, isolation steps of a subpopulation before analysis could be required. This may add a layer of complexity in carrying out real-time monitoring. The new method, nevertheless, can be extended to other diseases, for example, Alzheimer's, cardiovascular abnormality, and infectious diseases due to many potential merits compared to the conventional tissue biopsy.

Although continuous glucose monitoring (CGM) is the only approved real-time biomarker detection to date ${ }^{4}$, unattended care of patient will be realized in a hospital or even at home in the near future, e.g., 2020s. However, many hurdles exist as

Department of Biotechnology and Bioinformatics, Korea University, 2511 Sejong-ro, Sejong 30019, Republic of Korea

*Correspondence and requests for materials should be addressed to S.H. Paek (『shpaek@korea.ac.kr) mentioned, for example, bulky label-free sensor system, frequent withdrawal of blood without infection, and continual separation of blood cells. For the time being, this may restrict the use of real-time monitoring techniques within a medical care unit. Approximately 30 years were spent until the CGM system was introduced on the market though using a single enzyme reaction. Various technologies developed for CGM are expected to speed up the commercial launch of the binding reaction-based continuous sensor in a relatively short period of time (e.g., 10 years).

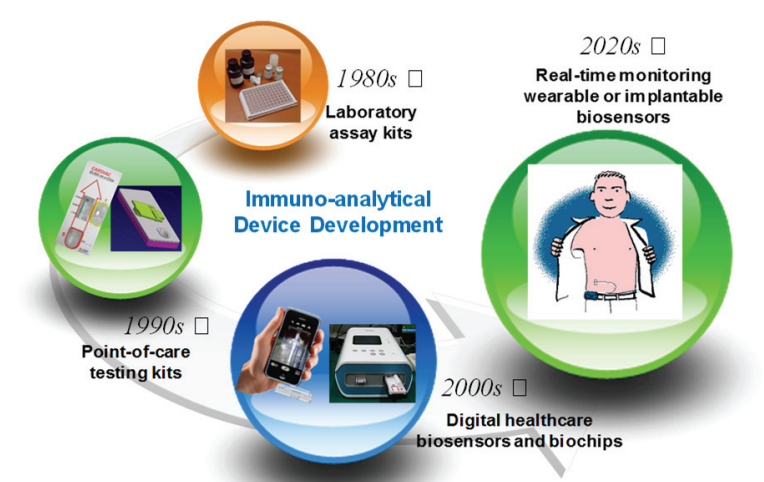

Figure 1. Historical view of immuno-analytical device technologies.

\section{References}

1. Rogers, M.L. \& Boutelle, M.G. Real-time clinical monitoring of biomolecules. Annu. Rev. Anal. Chem. 6, 427453 (2013).

2. Kim, D.H., Cho, I.H., Park, J.N., Paek, S.H., Cho, H.M. \& Paek S.H. Semi-continuous, real-time monitoring of protein biomarker using a recyclable surface plasmon resonance sensor. Biosens. Bioelectr. 88, 232-239 (2017).

3. Palmirotta, R., Lovero, D., Cafforio, P., Felici, C., Mannavola, F., Pellè, E., Quaresmini, D., Tucci, M. \& Silvestris, F. Liquid biopsy of cancer: a multimodal diagnostic tool in clinical oncology. Ther. Adv. Med. Oncol. 10, 1758835918794630 (2018).

4. The Juvenile Diabetes Research Foundation Continuous Glucose Monitoring Study Group. Continuous glucose monitoring and intensive treatment of type 1 diabetes. $N$. Engl. J. Med. 359, 1464-1476 (2008). 